\title{
Proteinuria e trapianto di rene
}

\author{
V. Sparacino, A. Amato
}

UO di Nefrologia $2^{\text {a }}$ con Emodialisi e Trapianto, Centro Trapianti di Rene "L. Sciascia”, ARNAS Ospedale

Civico, Palermo

\section{Introduzione}

La proteinuria è una delle principali anomalie urinarie ed è un esame basilare nella pratica clinica del nefrologo. Il trapianto renale non fa eccezione a questa regola generale.

Il glomerulo renale intatto, in virtù della sua struttura anatomica e, in particolar modo, delle proprietà dei podociti e delle loro giunzioni, costituisce una barriera efficientissima al passaggio di proteine nello spazio urinario durante il processo di ultrafiltrazione (1). Le poche proteine che sono filtrate (albumina in minima quantità e altre proteine a basso peso molecolare come la Beta2microgloblina, l'alpha1-macroglobulina, la proteina di Tamm-Horshfall e la Retinol Binding Protein) sono riassorbite prontamente a livello del tubulo prossimale: di conseguenza, la perdita "normale" di proteine nelle urine di un soggetto sano è virtualmente nulla. In realtà l'impiego in laboratorio di tecniche di determinazione sufficientemente sensibili ha consentito di riconoscere che esiste una fisiologica perdita di proteine nelle urine che ammonta a meno di $80 \mathrm{mg} /$ die di proteine totali e a meno di $20 \mathrm{mg} /$ die di albumina. Una albuminuria superiore ai $30 \mathrm{mg} /$ die fino ai $300 \mathrm{mg} /$ die rientra nella definizione corrente di "microalbuminuria" e costituisce un segnale precoce di incipiente danno renale, nonché un indicatore statisticamente potente di rischio cardiovascolare, in particolare nei pazienti diabetici, ipertesi, obesi o con altre combinazioni di fattori di rischio cardiovascolari. Le proteinurie superiori a questo limite sono storicamente definite proteinurie "macroscopiche" e sono associate a patologia renale conclamata; la proteinuria nefrosica ( $>3-3,5 \mathrm{~g} / \mathrm{die}$ ) è poi da considerasi "per definizione" espressione di patologia glomerulare.

Il trapianto renale fornisce generalmente al paziente un patrimonio di nefroni inferiore rispetto alla dotazione "fisiologica" prevista dalla natura; questo verosimilmente porta per sé all'attivazione dei meccanismi di compenso descritti nell'oramai classico modello del "nefrone intatto". Il rene trapiantato, inoltre, è esposto a una serie di fattori di potenziale danneggiamento: in primo luogo $\mathrm{i}$ tradizionali fattori di rischio cardiovascolare (ipertensione, diabete, alterazioni del metabolismo lipidico); in secondo luogo i fattori di rischio propri dei pazienti con insufficienza renale (alterazioni del metabolismo calciofosforo, anemia); infine a fattori di rischio unici del trapianto renale che sono le potenziali lesioni nella fase di prelievo e di ischemia-riperfusione, l'effetto della convivenza con il sistema immunitario del paziente trapiantato e l'effetto dei farmaci immunosoppressivi, che può essere di tossicità diretta sul rene o mediata attraverso gli altri fattori di rischio di cui sopra o le infezioni.

Alla luce di quanto prima esposto, è importante precisare che, poiché le nostre conoscenze sulla proteinuria sono basate sostanzialmente sull'esperienza nel rene nativo, l'estrapolazione di queste e la loro applicazione al trapianto renale deve necessariamente tenere presente le peculiarità di quest'ultimo.

\section{Misurazione della proteinuria}

La raccolta delle urine delle 24 ore rappresenta ancora oggi il "gold standard" della determinazione della proteinuria. Tuttavia, come noto, essa pone dei limiti pratici che hanno portato molti Centri Nefrologici ad adottare metodiche alternative che utilizzano un campione di urine "spot", come il rapporto urinario albumina/creatinina o proteine/creatinina. Quest'ultime metodiche hanno avuto una enorme diffusione e sono state validate scientificamente, pur non essendo immuni anch'esse da inconvenienti ed imprecisioni (2).

Per il trapianto renale, non esistendo al momento indicazioni specifiche, è ragionevole che i nefrologi continuino a utilizzare lo stesso approccio impiegato per il rene nativo. 


\section{Prevalenza della proteinuria nel trapianto renale}

Lo studio della prevalenza della proteinuria nei pazienti con trapianto di rene è essenziale per comprenderne il significato clinico.

A differenza di quanto accade nella popolazione generale, dove, come discusso, la proteinuria è infrequente ed è quasi invariabilmente da considerarsi segno di patologia renale, nel trapianto di rene la proteinuria è un riscontro molto comune e, pertanto, la sua interpretazione diviene assai meno ovvia che nel rene nativo.

Una serie di studi ha valutato la prevalenza di proteinuria in soggetti con trapianto renale, riportando stime piuttosto variabili (dal 7,5 al 45\%) (3). Questa cospicua variabilità sembra in buona parte riflettere alcuni fattori metodologici, in primo luogo il diverso livello di proteinuria utilizzato come soglia per stabilirne la significatività. Prevedibilmente, infatti, le casistiche che utilizzavano un valore di cut-off $>1 \mathrm{~g} /$ die registravano una prevalenza intorno al 15\%; di contro gli studi dove si utilizzavano criteri sovrapponibili a quelli del rene nativo $(>0,15-0,5$ $\mathrm{g} / \mathrm{die}$ ) riportavano una prevalenza maggiore.

Negli studi di Sancho e Halimi la percentuale di pazienti trapiantati con proteinuria $>0,5 \mathrm{~g} /$ die era rispettivamente del 20 e del 35\% $(4,5)$. Nel lavoro di Amer, quasi la metà dei pazienti $(45 \%)$ era proteinurico a 12 mesi dal trapianto usando un valore soglia analogo a quello dei soggetti non trapiantati $(0,15 \mathrm{~g} / \mathrm{die})(6)$, molto significativamente, di questo $45 \%$, i due terzi circa avevano una perdita di "basso grado" compresa tra 0,15 e 0,5 g/die. In generale dunque, adoperando i criteri validi per il rene nativo, il riscontro di proteinuria è comune nel trapianto renale.

\section{Fattori di rischio e valore prognostico della proteinuria post-trapianto}

La comparsa di proteinuria nel post-trapianto è stata correlata a diversi fattori di rischio. Alcuni autori hanno trovato una relazione con le caratteristiche dell'organo (età avanzata del donatore, causa di morte cardiovascolare, tempo di ischemia prolungato) (7), altri invece hanno descritto un rapporto con fattori relativi al ricevente: pressione arteriosa, creatinina plasmatica, episodi di rigetto acuto e ritardo di ripresa funzionale dell'organo. Tuttavia, gran parte dei lavori che hanno studiato queste correlazioni, sebbene pervengano a conclusioni plausibili e teoricamente convincenti, non sono del tutto ineccepibili dal punto di vista metodologico e dell'analisi statistica (3).
Per quanto riguarda invece il valore prognostico della proteinuria, le informazioni disponibili in letteratura sono concordi nel definire che, analogamente a quanto accade nel rene nativo, la presenza e l'entità della proteinuria sono fattori prognostici negativi in termini di sopravvivenza sia del trapianto sia del paziente.

In diversi lavori, dove l'analisi veniva aggiustata per fattori confondenti quali la pressione arteriosa e la funzione renale, la presenza di proteinuria aumentava mediamente di due-tre volte il rischio di perdita della funzione del trapianto renale e fino a due volte quello di mortalità; nello studio di Amer si stimava un aumento del rischio di perdita del trapianto del $27 \%$ per ogni aumento di un grammo/die della proteinuria (6). Non è noto se il valore prognostico negativo della proteinuria nel trapianto renale derivi dal fatto che essa esprime la presenza di un danno renale o se la proteinuria per sé abbia, come dimostrato nel rene nativo, un effetto diretto di ulteriore danneggiamento del rene.

\section{Istopatologia nei pazienti trapiantati con proteinuria}

Alcuni autori hanno esplorato la correlazione tra proteinuria ed alterazioni istopatologiche del trapianto renale. Anche in questo caso, i risultati ottenuti dipendono dall'entità della proteinuria al momento della biopsia renale.

I lavori di First e Yakupoglu $(8,9)$ hanno esaminato i dati istopatologici di 77 e 73 pazienti rispettivamente che venivano biopsiati in presenza di una importante proteinuria ( $>2-3 \mathrm{~g} / \mathrm{die})$. Entrambi i lavori, benché eseguiti in epoca diversa, hanno coerentemente dimostrato che nella stragrande maggioranza dei casi (oltre il 75\%) la diagnosi istologica era di patologia a livello glomerulare, sia sotto forma di recidiva della patologia glomerulare di base sia sotto forma di glomerulonefriti ex-novo, inclusa la glomerulopatia da trapianto, entità istopatologica esclusiva e tipica del trapianto renale (10).

La già citata casistica di Amer (6) è ancora più interessante, perché presenta i risultati di un numero considerevole di pazienti (276) in cui la biopsia renale veniva eseguita "di protocollo" a un anno dal trapianto, cioè prescindendo da qualunque indicazione clinica di qualsivoglia natura. Analogamente a quanto già evidenziato in precedenza, i soggetti che avevano al momento della biopsia una proteinuria superiore al grammo e mezzo (meno del 10\% dei pazienti inclusi nello studio, come già detto nel paragrafo precedente) avevano generalmente alterazioni istologiche a livello glomerulare. Da precisare però che, per converso, non tutti i pazienti con segni 
istologici di patologia glomerulare avevano necessariamente una proteinuria importante, per cui si può dedurre che la presenza di una proteinuria cospicua è altamente suggestiva di interessamento glomerulare, ma le glomerulopatie non possono essere escluse in soggetti con proteinuria modesta.

In ogni caso, i numerosi soggetti (30-35\% dei pazienti inclusi nello studio) con proteinuria di basso grado $(0,15-$ $0,5 \mathrm{~g} / \mathrm{die}$ ) tendevano per lo più a presentare un aspetto bioptico normale o alterazioni aspecifiche indicative di nefropatia cronica del trapianto (CAN, chronic allograft nephropathy); fatto interessante, le biopsie con segni istologici di rigetto acuto tendevano in media ad appartenere a soggetti scarsamente proteinurici, così come le biopsie con altre diagnosi più rare quali la nefropatia da BK polioma virus.

\section{Trattamento della proteinuria}

La riduzione della proteinuria è uno degli obiettivi principali della terapia nefrologica per rallentare l'evoluzione delle nefropatie nel rene nativo. L'efficacia di questo intervento è supportata da una grande mole di evidenze; altrettanto consolidato è il ruolo del blocco farmacologico del sistema renina-angiotensina (RAS, renin-angiotensin system) nella riduzione della proteinuria e nella nefroprotezione in soggetti con insufficienza renale cronica $(11,12)$.

Sfortunatamente, in contrasto con quanto raccolto nel campo del rene nativo, nessun analogo corpo di evidenze è disponibile per il trapianto renale, nonostante $\mathrm{i}$ pazienti trapiantati presentino numerose buone ragioni teoriche per beneficiare di queste terapie (alta prevalenza di proteinuria, ridotto numero di nefroni, elevata mortalità cardiovascolare).

Una recente revisione sistematica sul blocco del RAS ha messo in evidenza che l'utilizzo degli AceI e dei sartanici determina in effetti una riduzione della proteinuria (13); tuttavia gli effetti sulla sopravvivenza del trapianto e del paziente sono molto controversi $(14,15)$. In questo momento l'impiego di terapie antiproteinuriche ed in particolare di AceI e sartanici nel trapianto renale, benché praticato da molti nefrologi per estrapolazione dall'esperienza sul rene nativo, non è di fatto basato su alcuna solida dimostrazione scientifica.

\section{Proteinuria da rene nativo}

Un numero crescente di pazienti ha oggi accesso al trapianto renale in presenza di una funzione renale residua e di una diuresi conservata (come nel caso del trapianto pre-emptive). In queste circostanze la presenza di una proteinuria nell'immediato post-trapianto, specie se cospicua, orienta verso la provenienza dal rene nativo. Come ben descritto da Mysniak (16), la proteinuria del rene nativo tende di regola a diminuire progressivamente nel corso delle settimane dopo il trapianto, così come la funzione renale residua medesima; pertanto, un andamento crescente della proteinuria nel tempo deve farne sospettare la provenienza dal trapianto renale.

\section{Proteinuria e terapia immunosoppressiva}

La correlazione tra proteinuria e terapia immunosoppressiva è divenuta un argomento clinicamente rilevante in seguito alla diffusione dei farmaci inibitori del mTOR (Sirolimus ed Everolimus). Questi farmaci si sono affacciati nel campo dellimmunosoppressione del trapianto renale carichi di aspettative: la mancanza di nefrotossicità rispetto ai più vecchi inibitori della calcineurina (CNI, ciclosporina e tacrolimus), l'azione favorevole sull'aterosclerosi e le malattie cardiovascolari e, infine, l'azione anti-tumorale. Sin dalle prime esperienze di conversione da CNI a mTORi, per arrivare poi ai moltissimi lavori pubblicati in letteratura su quest'argomento, fu osservata una tendenza alla comparsa o al peggioramento della proteinuria. Il fenomeno è particolarmente rilevante e prognosticamente sfavorevole in soggetti che presentano una proteinuria già significativa al momento della conversione o uno stadio avanzato di disfunzione renale da nefropatia cronica del trapianto (chronic allograft nephropathy: CAN). Sulle ragioni di questo effetto vi sono due principali interpretazioni, che peraltro non si escludono mutuamente: la prima è che la proteinuria nei regimi basati sugli mTORi compaia perché manca l'effetto antiproteinurico che i CNI esercitano in virtù dei loro effetti emodinamici sulla microcircolazione renale; la seconda è che, invece, gli mTORi possiedano un'azione diretta "tossica" a carico del rene ed in particolare dei podociti e del tubulo renale; quest'ultima tesi è avvalorata da diverse evidenze sperimentali $(17,18)$.

L'azione favorente la proteinuria degli mTORi ha portato a stabilirne delle indicazioni e dei criteri di impiego: ad esempio, nelle conversioni da CNI a mTORi, è consigliata la presenza di una proteinuria inferiore a $0,8-1 \mathrm{~g} / \mathrm{die}$, nonché la minimizzazione della dose del CNI piuttosto che la sua completa sospensione (19).

\section{Conclusioni}

La proteinuria è un riscontro molto comune nel trapianto renale.

Nella maggior parte dei casi si tratta di proteinurie di 
lieve entità $(<1 \mathrm{~g} /$ die) che si associano a danni aspecifici del tessuto renale, come nel caso della nefropatia cronica da trapianto. Questa tipologia di proteinuria è verosimilmente correlata a fattori di rischio quali l'età e le caratteristiche del donatore, il danno da ischemia-riperfusione e il danno immunologico e non immunologico che ha luogo nel ricevente. I farmaci inibitori del mTOR sono associati a proteinuria con più frequenza rispetto ai regimi immunosoppressivi basati su CNI, anche se la portata di questo effetto non è stata ancora chiarita.

La presenza di proteinuria importante aumenta la probabilità di trovare lesioni glomerulari, sia come recidiva della malattia di base, sia come patologia ex novo o, ancora, come patologia specifica del trapianto (glomerulopatia da trapianto). La individuazione di questi quadri è fondamentale perché presuppone specifiche misure terapeutiche. A prescindere dall'etiologia, la proteinuria è un fattore prognostico negativo sia per la sopravvivenza dell'organo sia per la sopravvivenza del paziente. L'impiego di terapie antiproteinuriche (come gli AceI e i Sartani) sulla scorta della loro documentata efficacia nella patologia del rene nativo, pur essendo comune e ragionevole nella pratica clinica, non è supportato da chiare evidenze.

\section{Indirizzo degli Autori:}

Vito Sparacino, MD

UO di Nefrologia $2^{\text {a }}$ con Emodialisi e Trapianto

Centro Trapianti di Rene "L. Sciascia"

ARNAS Ospedale Civico

Via Carmelo Lazzaro 2/A

90127 Palermo

vitosparacino@ospedalecivicopa.org

\section{Bibliografia}

1. Haraldsson B, Nystrom J, Deen WM. Properties of the Glomerular Barrier and Mechanisms of Proteinuria. Physiol Rev 2008; 88: 451-87.

2. Cirillo M. Evaluation of glomerular filtration rate and of albuminuria/proteinuria. J Nephrol 2010; 23: 125-32.

3. Knoll GA. Proteinuria in Kidney Transplant Recipients: Prevalence, Prognosis, and Evidence-Based Management. Am J Kidney Dis 2009; 54: 1131-44.

4. Sancho A, Gavela E, Avila A, et al. Risk factors and prognosis for proteinuria in renal transplant recipients. Transplant Proc 2007; 39: 2145-7.

5. Halimi JM, Laouad I, Buchler M, et al. Early low grade proteinuria: Causes, short-term evolution and long-term consequences in renal transplantation. Am J Transplant 2005; 5: 2281-8.

6. Amer H, Fidler ME, Myslak M, et al. Proteinuria after kidney transplantation, relationship to allograft histology and survival. Am J Transplant 2007; 7: 2748-56.

7. Halimi J, Laouad I, Buchler M, et al. Early proteinuria is a strong indicator of donor renal lesions, ischemia-reperfusion injury and immunological aggression._Transplant Proc 2006; 38: 2319-20.

8. First MR, Vaidya PN, Maryniak RK, et al. Proteinuria following transplantation. Correlation with histopathology and outcome. Transplantation 1984; 38: 607-12.

9. Yakupoglu U, Baranowska-Daca E, Rosen D, et al. Posttransplant nephrotic syndrome: A comprehensive clinicopathologic study. Kidney Int 2004; 65: 2360-70.

10. Fotheringham J, Angel CA, McKane W. Transplant glomerulopathy: morphology, associations and mechanism. Nephron Clin Pract 2009; 113: c1-7.

11. Sarafidis PA, Bakris GL. Renin-angiotensin blockade and kidney disease. Lancet. 2008; 372: 511-2.

12. Bakris GL. Slowing nephropathy progression: focus on proteinuria reduction. Clin J Am Soc Nephrol 2008; 3(Suppl 1): S3-10.

13. Hiremath S, Fergusson D, Doucette S, et al. Renin angiotensin system blockade in kidney transplantation: A systematic review of the evidence. Am J Transplant 2007; 7: 2350-60.

14. Heinze G, Mitterbauer C, Regele H, et al. Angiotensin converting enzyme inhibitor or angiotensin II type 1 receptor antagonist therapy is associated with prolonged patient and graft survival after renal transplantation. J Am Soc Nephrol 2006; 17: 889-99.

15. Opelz G, Zeier M, Lau G, et al. No improvement of patient or graft survival in transplant recipients treated with angiotensin-converting enzyme inhibitors or angiotensin II type 1 receptor blockers: A Collaborative Transplant Study Report. J Am Soc Nephrol 2006; 17: 3257-62.

16. Myslak M, Amer H, Morales $\mathrm{P}$, et al. Interpretino posttransplant proteinuria in patients with proteinuria pretransplant. Am J Transplant 2006; 6: 1660-5.

17. Biancone L, Bussolati B, Mazzucco G, et al. Loss of nephrin expression in glomeruli of kidney-transplanted patients under m-TOR inhibitor therapy. Am J Transplant 2010; 10: 2270-8.

18. Franz S, Regeniter A, Hopfer H, et al. Tubular toxicity in sirolimus- and cyclosporine-based transplant immunosuppression strategies: an ancillary study from a randomized controlled trial. Am J Kidney Dis 2010; 55: 335-43. Epub 2009 Nov 17.

19. Schena FP. Review of symposium. Sirolimus in kidney transplantation. Transplantation. 2009; 27; 87(Suppl 8): S30-3. 